\title{
Enhancing network transmission capacity by efficiently allocating node capability
}

\author{
Guo-Qing Zhang, Shi Zhou, Di Wang, Gang Yan, and Guo-Qiang Zhang *广
}

October 13, 2009

\begin{abstract}
A network's transmission capacity is the maximal rate of traffic inflow that the network can handle without causing congestion. Here we study how to enhance this quantity by redistributing the capability of individual nodes while preserving the total sum of node capability. We propose a practical and effective node-capability allocation scheme which allocates a node's capability based on the local knowledge of the node's connectivity. We show the scheme enhances the transmission capacity by two orders of magnitude for networks with heterogenous structures.

Keywords:Networks, transmission capacity, traffic flow simulation, network modeling, network topology, betweenness.
\end{abstract}

\section{Introduction}

There are different ways to enhance a network's transmission capacity. A number of routing strategies have been introduced to route traffic based on

\footnotetext{
${ }^{*}$ This work is supported by the National Natural Science Foundation of China under grant no. 60673168, the Hi-Tech Research and Development Program of China under grant no. 2006AA01Z207 and the China Next Generating Internet Project under grant no. CNGI04-7-1D. S. Zhou is supported by The Royal Academy of Engineering and EPSRC (UK) under grant no. 10216/70.

${ }^{\dagger}$ G.-Qing Zhang, D. Wang and G.-Qiang Zhang are with Institute of Computing Technology, Chinese Academy of Sciences (CAS), and Graduate University of CAS, Beijing, 100190, P. R. China (e-mail:gqzhang@ict.ac.cn, guoqiang@ict.ac.cn). S.Zhou is with Department of Computer Science, University College London, Malet Place, London, WC1E 6BT, United Kingdom (e-mail: s.zhou@cs.ucl.ac.uk). G. Yan is with University of Science and Technology of China, Hefei, 230026, P. R. China.
} 
the topological properties of the network [1, 2] or the real-time load distribution on links [3]. Recently we reported [4] that a network's transmission capacity can be increased by removing a few links with certain topological properties. These work assume that all nodes or links are assigned with uniform resources. However this is not the case in many networks and it is often difficult to change a network's routing protocol or topology.

One can also enhance a network's transmission capacity by simply increasing the resources of all nodes or links. It is, however, very expensive. A more realistic and economical approach is to redistribute the resources in the network, such that those that handle higher volumes of traffic load have more resources whereas those that handle less load have fewer resources. This should be done under the condition that the total amount of resources allocated in the network is fixed. This is relevant to the design and engineering of communication networks where a key goal is to deploy limited resources in a way to achieve the best network performance.

Node capability and link bandwidth are two major resources to be allocated. Whether to redistribute the node capability or the link bandwidth depends on what is the major cause for congestion in a network. For example when a new generation of routers are deployed, the processing power of routers are greatly improved, then the congestion is mainly caused by the lack of link bandwidth; whereas when optical fibre replaced cable, link bandwidth increased by a number of magnitudes, then the congestion is mainly caused by the lack of node processing power. Over the time these two situations may happen alternately in communication networks. In this paper we focus on the allocation of node capability for those networks where links have sufficient bandwidth. In our future work we will study the redistribution of link bandwidth.

This paper is organised as follows. In Section II we introduce three typical communication network topology models: a random graph [5], a scale-free graph [6] and an Internet-like graph [7]. We also present a simple yet widely used traffic-flow model based on the shortest-path routing [8]. In Section III we study a number of degree-based node-capability allocation schemes using simulations based on the traffic-flow and topology models. We introduce a scheme which enhances a network's transmission capacity by two orders of magnitude by allocating a node's capability as a power function of the node's connectivity. In Section IV we discuss an alternative way to estimate the optimal power exponent used in our scheme, and compares our scheme with a previous scheme [8] which is based on the topological property of betweenness [9]. 
Table 1: Network properties and simulation results

\begin{tabular}{lccc}
\hline \hline Topology property & ER & BA & PFP \\
\hline Number of nodes & 4,000 & 4,000 & 4,000 \\
Number of links & 12,000 & 12,000 & 12,000 \\
Degree distribution & Poisson & $\propto k^{-3}$ & $\propto k^{-2.2}$ \\
Maximum degree & 18 & 156 & 979 \\
Average shortest distance & 4.82 & 4.17 & 3.12 \\
Average clustering coef. & 0.001 & 0.007 & 0.253 \\
\hline Node-capability scheme & Critical package-generating rate $\lambda_{c}$ \\
\hline$C \propto 1$ & $885_{ \pm 21}$ & $57_{ \pm 17}$ & $48_{ \pm 9}$ \\
$C \propto k$ & $2,616_{ \pm 82}$ & $1,289_{ \pm 65}$ & $4,419_{ \pm 108}$ \\
$C \propto k^{1.5}$ & $4,319_{ \pm 117}$ & $2,954_{ \pm 302}$ & $1,636_{ \pm 83}$ \\
$C \propto k^{\alpha^{*}}$ & $4,319_{ \pm 117}$ & $3,284_{ \pm 241}$ & $5,126_{ \pm 177}$ \\
$C \propto B$ & $6,576_{ \pm 215}$ & $7,604_{ \pm 184}$ & $11,592_{ \pm 204}$ \\
\hline Optimal exp. $\alpha^{*}$ in Fig. 1 & $1.50_{ \pm 0.05}$ & $1.40_{ \pm 0.03}$ & $1.10_{ \pm 0.03}$ \\
Fitting exp. $\alpha^{\prime}$ in Fig. 2 & $1.49_{ \pm 0.03}$ & $1.37_{ \pm 0.03}$ & $1.11_{ \pm 0.02}$ \\
\hline \hline
\end{tabular}

\section{Background}

We consider three network models as examples of typical topologies of computer and communication networks, which are the Erdös-Rényi (ER) model [5], the Barabási-Albert (BA) model [6] and the positive-feedback preference (PFP) model [7]. In graph theory, degree $k$ is defined as the number of links a node has. The ER model generates random networks with a Poisson degree distribution, where most nodes have a degree close to the average degree. The ER model has been used to describe the structure of LAN and wireless ad hoc networks. The BA model generates the so-called 'scale-free' networks with a power-law degree distribution, where a few nodes have very large degrees and the majority nodes have only a few links. Many communication networks are found to be scale-free [10] and the BA model has been used to study the error and attack tolerance of such networks [11. The PFP model generates a network structure which is very similar to the Internet at the autonomous systems level [7, 12]. For each model we generate ten networks using random seeds to the same numbers of nodes and links. Table 1 shows properties of the three models.

In this study we adopt a similar traffic-flow model used in [1, 4, 8, 13. For a network with $N$ nodes, at each time step, $\lambda$ packets are generated at randomly selected nodes. The destination is chosen randomly. A packet 
is routed following the shortest path between source and destination. The shortest-path routing strategy is widely used in communication networks, such as the Open Shortest Path First (OSPF) routing protocol. A node $i$ is assigned a capability, $C_{i}$, which is the maximal number of packets the node can handle at a time step. When the total number of arrived and newly created packets is larger than $C_{i}$, the packets are stored in the node's queue and will be processed in the following time steps on a first-in-first-out (FIFO) basis. If there are several shortest paths for one packet, one is chosen randomly. Packets reaching their destination are deleted from the system. As in 1, 4, 8, 13, node buffer size in this traffic-flow model is set as infinite as it is not relevant to the occurrence of congestion.

For small values of the packet-generating rate $\lambda$, the number of packets on the network is small so that every packet can be processed and delivered in time. Typically, after a short transient time, a steady state for the traffic flow is reached where, on average, the total numbers of packets created and delivered are equal, resulting in a free-flow state. For larger values of $\lambda$, the number of packets created is more likely to exceed what the network can process in time. In this case traffic congestion occurs. As $\lambda$ is increased from zero, we expect to observe two phases: free flow for small $\lambda$ and a congested phase for large $\lambda$, with a phase transition from the former to the latter at the critical packet-generating rate $\lambda_{c}$.

In order to measure $\lambda_{c}$, we use the order parameter [14] $\eta=\lim _{t \rightarrow \infty} \frac{\langle\Delta \Theta\rangle}{\lambda \Delta t}$, where $\Theta(t)$ is the total number of packets in the network at time $t, \Delta \Theta=$ $\Theta(t+\Delta t)-\Theta(t)$, and $\langle\cdots\rangle$ indicates the average over time windows of $\Delta t$. For $\lambda<\lambda_{c}$ the network is in the free-flow state, then $\Delta \Theta \approx 0$ and $\eta \approx 0$; and for $\lambda>\lambda_{c}, \Delta \Theta$ increases with $\Delta t$ thus $\eta>0$. Therefore in our simulation we can determine $\lambda_{c}$ as the transition point where $\eta$ deviates from zero.

\section{Enhancing network transmission capacity}

The critical packet-generating rate $\lambda_{c}$ is used to measure a network's overall transmission capacity, which is the maximal amount of traffic flow that a network can handle without causing congestion. Increasing network transmission capacity is one of the major goals for network design and engineering.

In the following we study a number of degree-based node-capability allocation schemes. For each network model, we obtain the network's critical packet-generating rate $\lambda_{c}$ by running the traffic-flow simulation on the ten instance networks of the model. Table 1 shows the average and the bounds of $\lambda_{c}$ for different node-capability allocation schemes. For comparison pur- 
pose without losing generality, we keep the sum of node capability in all simulations the same, i.e. $\sum_{i} C_{i}=\sum_{i} k_{i}=2 L$, where $L$ is the number of links.

The first scheme is a simplistic baseline case where we assign all nodes with a uniform capability, i.e. $C$ is equal to the average degree $\langle k\rangle$ (shown in Table 1 as $C \propto 1$ ). The second scheme is to allocate a node's capability proportional to its degree $k$, i.e. $C \propto k$. The underlying heuristic is that the larger degree (i.e. more incoming traffic from neighbors), the more traffic a node needs to handle. Table 1 shows there is a substantial increase of $\lambda_{c}$ for all the networks when node capability allocation changes from uniform scheme to degree-proportional scheme. For the ER network, $\lambda_{c}$ increases three times while for the PFP model the increase is two orders of magnitude. This result highlights the importance of respecting the difference in node degrees when allocating node capability. This is particularly true for networks featuring a heterogenous structure where node degrees vary hugely, such as the BA and PFP models.

In the third scheme we further increase the weight of node degree by assigning a node's capability proportional to its degree raised to the power of 1.5, i.e. $C \propto k^{1.5}$. As shown in Table 1, this scheme produces better results for the ER and BA networks, but it overdoes for the PFP network. This is because the PFP network has a few nodes with disproportionably large degrees (see the maximum degree in Table 1). These nodes, although a small number, take too large a share of the total node capability. This leaves the majority of nodes, which are poorly connected, with very little capability and therefore restrains the network overall transmission capacity. This suggests there is an optimisation problem as how to achieve the balance between individual and collective interest.

In order to examine this issue systematically, we define a generic degreebased scheme as $C \propto k^{\alpha}$. When $\alpha=0$ it is the uniform scheme and when $\alpha=1$ it is the degree-proportional scheme. Fig. 1 shows the critical packagegenerating rate $\lambda_{c}$ as a function of the exponent $\alpha$. For each network the $\lambda_{c}$ peaks at a characteristic value of $\alpha^{*}$, which is the optimal exponent for the degree-based scheme. Table 1 gives the value of $\alpha^{*}$ and the peak value of $\lambda_{c}$ when using the node-capability allocation scheme $C \propto k^{\alpha^{*}}$.

\section{Discussion and Conclusion}

For any network topology we can obtain $\alpha^{*}$ by running the traffic-flow simulation as described above, which, however, is time-consuming. Here we 


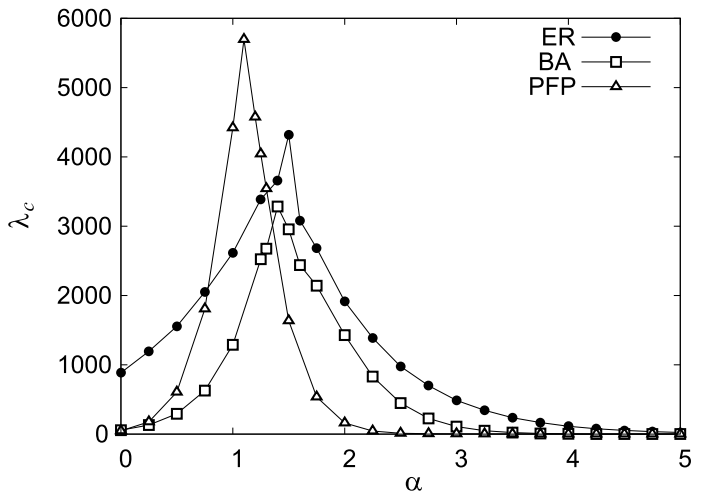

Figure 1: Critical packet-generating rate $\lambda_{c}$ as a function of exponent $\alpha$.

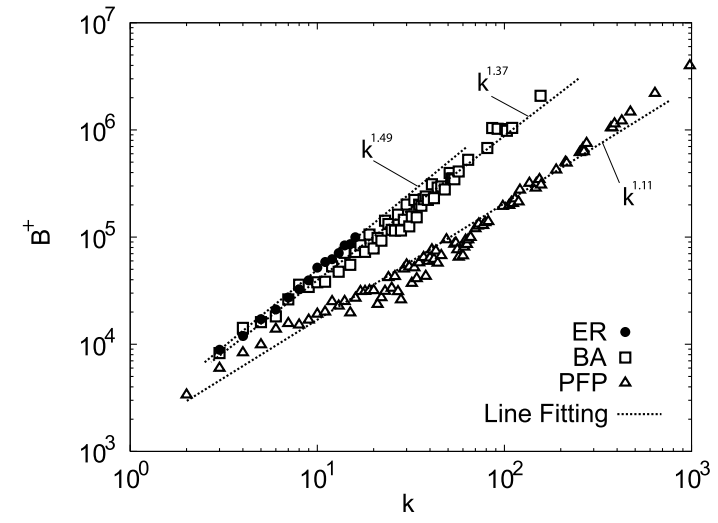

Figure 2: Largest betweenness $B^{+}$of $k$-degree nodes. The line fitting is obtained by least square fitting technique. 
introduce an alternative way to estimate $\alpha^{*}$ without having to run the simulation. Recently we analytically proved that a network's critical packetgenerating rate can be estimated as $\lambda_{c}=\min _{i \in V}\left\{\frac{C(i) N(N-1)}{B(i)}\right\}$, where $V$ is the set of node indexes [15] and $B(i)$ is the betweenness of node $i$ (see definition in [9]). For the node-capability allocation scheme of $C \propto k^{\alpha^{*}}$ we have $\lambda_{c} \propto \min _{k \in K}\left\{\frac{k^{\alpha^{*}} N(N-1)}{B^{+}(k)}\right\}$ where $K$ is the set of possible degree values and $B^{+}(k)$ is the largest betweenness value of $k$-degree nodes. (We use the largest betweenness because $\lambda_{c}$ is constrained by the largest traffic load a $k$-degree node has.) This suggests that for our scheme to produce a sound result, $k^{\alpha^{*}}$ should approximate $B^{+}(k)$. As shown in Fig. 2 this is indeed the case where $B^{+}(k) \propto k^{\alpha^{\prime}}$ and $\alpha^{\prime} \simeq \alpha^{*}$ (see Table 1 ). This provides a convenient way to estimate $\alpha^{*}$ by fitting the function of $B^{+}(k)$. If a network has a well-defined model, the betweenness calculation can be simplified by computing on a smaller graph generated by the model.

A previous work [8] suggested that $\lambda_{c}$ is maximised by allocating a node's capability according to the node's betweenness. As shown in Table 1 this scheme indeed produces better results. This is because in the traffic-flow model, betweenness precisely estimates the traffic load at each node. This scheme, however, faces several practical issues. Firstly, betweenness is sensitive to topology changes. A minor change of a network's topology, e.g. adding a new link, could significantly alter the betweenness value of nodes in all parts of the network. This requires frequent recalculation of node betweenness for networks with non-static topologies. Secondly, betweenness calculation requires global knowledge of a network's topology, which is not often possible in practice. Finally, betweenness calculation, the dominant factor of this scheme's processing complexity, is not a trivial task, especially for large networks. Therefore, the betweenness scheme is mainly used to give the theoretical upper bound of $\lambda_{c}$ for evaluation purpose. While in practice it can only be used for networks with static topologies, or when the requirement on network transmission capacity is so high that the benefit of applying this scheme overruns the cost. By comparison our degree-based scheme $C \propto k^{\alpha^{*}}$ is more practical and robust. Firstly, we allocate a node's capability based on the local knowledge of node degree. Secondly, the value of $\alpha^{*}$ is determined by a network's macroscopic structure which is not sensitive to minor topology changes, so it can be reused. Finally, for networks with well-defined model, $\alpha^{*}$ can be estimated by doing the fitting on a much smaller network generated by the model, avoiding betweenness calculation for large real networks, which is also the dominator factor of this scheme's processing complexity. 
In summary we recommend our node-capability allocation scheme of $C \propto k^{\alpha^{*}}$ which can enhance a network's transmission capacity substantially without increasing the total amount of node capability. Usually, the value of $\alpha^{*}$ can be obtained by fitting the function of $B^{+}(k)$ without the need to run the traffic-flow simulation. Our scheme can be viewed as an approximation of the betweenness scheme with improved practicality and robustness.

\section{References}

[1] G. Yan, T. Zhou, B. Hu, Z.-Q. Fu, and B.-H. Wang, "Efficient routing on complex networks," Phys. Rev. E, vol. 73, p. 046108, 2006.

[2] B. Fortz and M. Thorup, "Internet Traffic Engineering by Optimizing OSPF Weights," Proc. of IEEE INFOCOM, 2000.

[3] J. Scholz, W. Krause and M. Greiner, "Decorrelation of networked communication flow via load-dependent routing weights," Physica A, vol. 387, pp. 2987-3000, 2008.

[4] G.-Q. Zhang, D. Wang, and G.-J. Li, "Enhancing the transmission efficiency by edge deletion in scale-free networks," Phys. Rev. E, vol. 76, p. 017101, 2007.

[5] P. Erdös and A. Rényi, "On random graphs," Publ. Math. Debrecen, vol. 6, p. 290, 1959.

[6] A. Barabási and R. Albert, "Emergence of scaling in random networks," Science, vol. 286, p. 509, 1999.

[7] S. Zhou and R. J. Mondragón, "Accurately modelling the Internet topology," Phys. Rev. E, vol. 70, no. 066108, 2004.

[8] L. Zhao, Y.-C. Lai, K. Park, and N. Ye, "Onset of traffic congestion in complex networks," Phys. Rev. E, vol. 71, p. 026125, 2005.

[9] K. I. Goh, B. Kahng, and D. Kim, "Universal behavior of load distribution in scale-free networks," Phys. Rev. Lett., vol. 87, no. 278701, p. 278701, 2001.

[10] M. Faloutsos, P. Faloutsos, and C. Faloutsos, "On power-law relationships of the Internet topology," Comput. Commun. Rev., vol. 29, pp. 251-262, 1999. 
[11] R. Albert, H. Jeong, and A. Barabasi, "Error and attack tolerance of complex networks," Nature, vol. 406, p. 378, 2000.

[12] S. Zhou, G.-Q. Zhang, and G.-Q. Zhang, "Chinese Internet AS-Level Topology," IET Commun., vol. 1, no. 2, pp. 209-214, 2007.

[13] B. Danila, Y. Yu, J. A. Marsh, and K. E. Bassler, "Optimal transport on complex networks," Phys. Rev. E, vol. 74, p. 046106, 2006.

[14] A. Arenas, A. Díaz-Guilera, and R. Guimerà, "Communication in networks with hierarchical branching," Phys. Rev. Lett., vol. 86, p. 3196, 2001.

[15] G.-Q. Zhang, B. Yuan, and G.-Q. Zhang, "Towards a comprehensive understanding of routing on complex networks", Proc. of 3rd EuroNGI Conf. on Next Generation Internet Networks, pp. 151-158, 2007. 